\title{
Anticoagulation for Atrial Fibrillation in Older Adults-Using Big Data for Big Questions
}

\author{
Terence J. Quinn ${ }^{1} \quad$ Deirdre A. Lane ${ }^{2}$ \\ ${ }^{1}$ Institute of Cardiovascular and Medical Sciences, University of \\ Glasgow, Glasgow, United Kingdom \\ ${ }^{2}$ Liverpool Centre for Cardiovascular Science, University of Liverpool \\ and Liverpool Heart \& Chest Hospital, Liverpool, United Kingdom
}

Thromb Haemost 2019;119:855-857.

Atrial fibrillation (AF) is predominantly a disease of older age, with a doubling of risk for each decade lived. ${ }^{1}$ As well as experiencing more $\mathrm{AF}$, older adults are more likely to suffer the consequences of $\mathrm{AF}$, most notably stroke, and they are more likely to be left dead or disabled following an AF-related stroke. ${ }^{1}$ Despite this age-associated 'treble whammy', the majority of evidence around treatment of AF has focused on middle-aged adults. This apparent disconnect between evidence and practice may be one reason why there is marked variation in clinical practice around treating $\mathrm{AF}$ in older adults. ${ }^{2}$

Of course age is just a number and even within the oldest old, there will be a spectrum of health from robust, community-dwelling through to frail, multi-morbid older adults. It is in this latter group that clinical trial evidence is especially lacking. $\mathrm{AF}$ is often associated with frailty and comorbidity. ${ }^{3}$ Yet, traditional, large cardiovascular randomised controlled trials (RCTs) have generally excluded such patients. Certainly, including older adults living with frailty in AF-based RCTs poses challenges, for example, there will be issues with capacity, attrition, test burden and competing risk. ${ }^{4}$ Encouragingly, there are emerging examples of trials that focus on frailty and also examples of trials that describe frailty syndromes as study outcomes. ${ }^{4}$

A potential solution to the perceived lack of generalisability of RCTs is to use existing clinical data from large registries and databases. Many such studies have been published, that have examined treatment patterns and outcomes. ${ }^{5-7}$ Secondary analysis of big data from real-world settings offers several advantages, not least that clinical hypotheses can be tested at scale for a fraction of the cost, time and manpower required for a prospective study. ${ }^{8}$ In this issue of Thrombosis \& Haemostasis, there are several papers where real-world data are used to improve our understanding of $\mathrm{AF}$ treatment in older adults. These papers are exemplars of what can be achieved through analysis of routine data, but they also highlight some of the limitations of the big data approach.

received

March 26, 2019

accepted after revision

March 26, 2019

Address for correspondence Deirdre A. Lane, PhD, Liverpool Centre for Cardiovascular Science, Institute of Ageing and Chronic Disease, University of Liverpool, William Henry Duncan Building, 6 West Derby Street, Liverpool, L7 8TX, United Kingdom (e-mail: deirdre.lane@liverpool.ac.uk).

Hohmann et $\mathrm{al}^{9}$ used information held in a German healthcare claims database to look at risk profiles of various anticoagulation treatment approaches in older adults. The dataset included 70,501 patients newly started on anticoagulation for $\mathrm{AF}(n=42,562$ [60.4\%] with non-vitamin $\mathrm{K}$ antagonist [VKA] oral anticoagulant [NOAC]). The population were categorised by age, frailty, co-morbidity and polypharmacy. Across all these categories, the risk of stroke and systemic embolism was higher than had been described in the seminal RCTs of NOACs. ${ }^{1}$ Comparing OAC treatments (NOAC vs.phenprocoumon), there was no signal of differential treatment efficacy between NOAC and a traditional VKA. However, when comparing safety profiles, in this case defined by major bleeding, there was a clear difference between groups in favour of NOAC prescribing.

Using a similar approach of interrogating a large German healthcare claims database, Hohnloser et $\mathrm{al}^{10}$ describe rates of OAC discontinuation and switching (VKA to NOAC, NOAC to VKA and NOAC to NOAC) for a cohort of older adults newly prescribed anticoagulation for AF. Again the cohort was impressively large, with 51,606 patients (30,138 [58.4\%] prescribed NOAC). We often think of anticoagulation for AF as a life-long treatment, however, in this cohort around one-third had discontinued anticoagulation at 1 year. Rates of discontinuation were similar across group, NOAC (29.5\%) and VKA (29.9\%). Older adults were more likely to persist with OAC; a reassuring finding given that this group are at higher risk of AFrelated stroke. In total, 5,149 (10\%) patients switched from their initial anticoagulant to another agent, with those initially prescribed VKA more likely to switch than those prescribed NOAC $(2,298$ [11\%] vs. 2,851 [9.5\%]). Change of OAC was associated with clinical events, both stroke and bleeding. There was also a temporal prescribing change with more conversion to NOAC over time, perhaps as physicians become more familiar with these new medications.

Finally, Field et al ${ }^{11}$ focused their attention on one of the outcomes of greatest concern to older adults, namely dementia.

(c) 2019 Georg Thieme Verlag KG Stuttgart · New York
DOI https://doi.org/ 10.1055/s-0039-1688482. ISSN 0340-6245. 
Using the U.K. Clinical Practice Research Database, they created matched cohorts with and without AF, totalling 91,372 (15,276 [16.7\%] with AF). By linking the primary care dataset to U.K. National Health Service secondary care registries, they examined incident dementia over an average of 2 years of follow-up. They report an increased risk between AF and incident dementia (adjusted hazard ratio:1.31 [95\% confidence interval:1.151.49]), albeit with a smaller effect than demonstrated in previous studies. ${ }^{12}$ By performing sub-group analyses looking at anticoagulation prescription and incident stroke, we can speculate on potential mechanisms underlying this association. Prescription of anticoagulation seemed to ameliorate the risk of dementia, suggesting that cardioembolism is likely to be a factor in any cognitive decline. However, the risk of dementia remained significant even after removing all those with stroke or transient ischaemic attack. This suggests that it is not only stroke that is driving cognitive change, or at least not clinically apparent stroke. A disheartening observation in this contemporary cohort was that around one-third of older adults with $\mathrm{AF}$ received no anticoagulation treatment.

So, what messages can we take from these three complementary analyses? Clearly, routinely collected data represent a powerful resource for research in older adults. The numbers included in these analyses would be almost impossible to replicate in a bespoke, prospective study. However, 'big data' are not necessarily better data and if the data have inherent limitations, the large sample size will only serve to make a biased estimate more precise. Of particular concern, are those patients who are not captured in the databases, for example, the two German studies only included AF patients newlyinitiating OAC. Yet, other data tell us that often the highest risk AF groups are not prescribed evidence-based therapies and so will not feature in these prescribing-based registers. ${ }^{2}$
The studies described looked at syndromes of older age through the lens of what is recorded and available in large databases. In this regard, co-morbidity and polypharmacy are relevant. However, for many older adults, the outcomes of interest may not be routinely recorded and clinical registerbased research may struggle to assess factors such as falls, disability and admission to care homes. Even where data are available, the validity is not guaranteed. Taking dementia as an example, recording of dementia in clinical registers tends to be specific but insensitive and may miss many living with the disease. $^{13}$ VKA medications are problematic for big data approaches, as the interpretation requires additional information on dosing and international normalised ratio, which is often lacking. In this regard, big data researchers will be glad of the increasing use of NOACs which are analytically easier to handle.

Even with the most sophisticated analyses, matching and adjustment, we must be cautious about how we interpret these observational data. Retrospective analyses can miss the nuance and clinical rationale for prescribing decisions and the preference for a particular anticoagulant strategy will not be made at random. This is likely to be a particular issue in older adults where the evidence to guide treatment decisions may be best practice recommendations rather than from RCTs. ${ }^{14}$

For all these reasons, big data approaches are best regarded as complementing other study methods including mechanistic pre-clinical experiments, bespoke cohorts and large-scale clinical trials (see - Fig. 1). Using routinely recoded data can generate new hypotheses to be tested or can validate results generated using other research methods. From the data presented in these three papers, we can be reasonably confident about prescribing NOACs in older adults but whether these drugs will reduce frailty syndromes such as dementia will require testing in large-scale trials. ${ }^{12}$

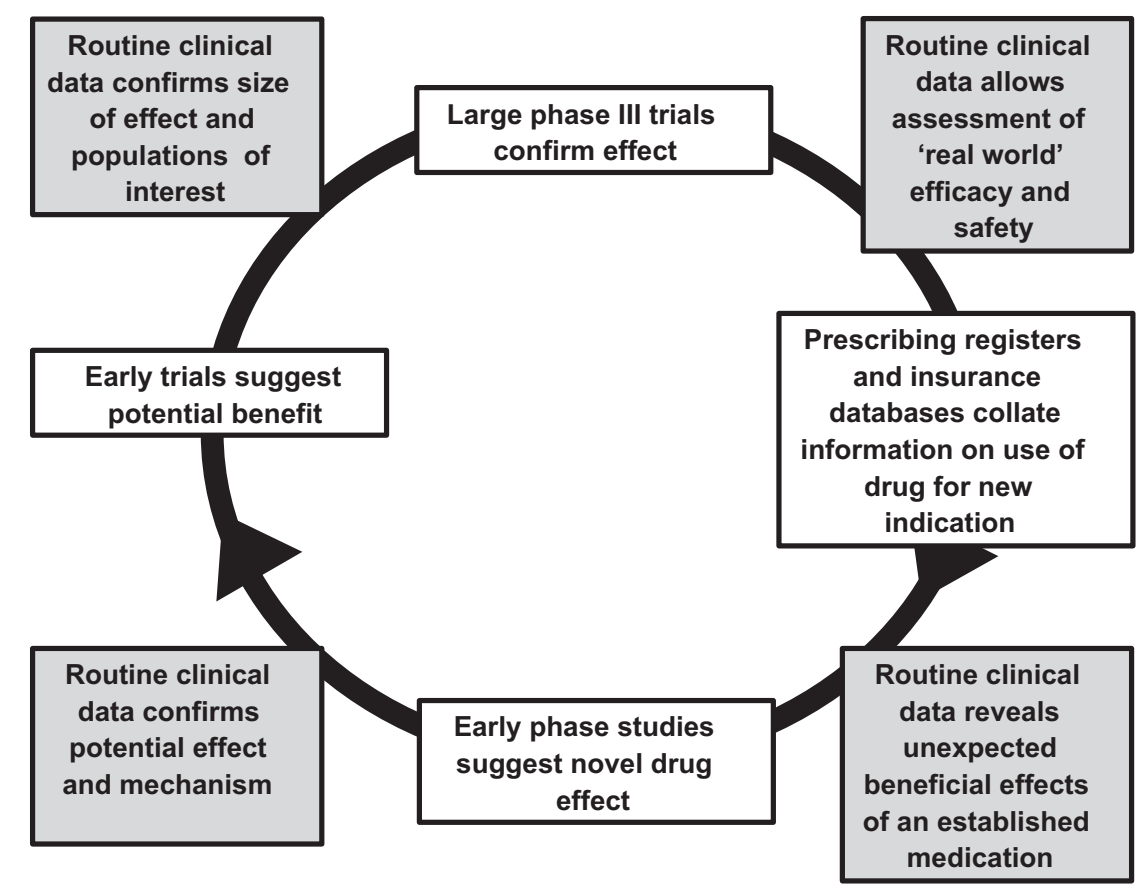

Fig. 1 Incorporating big data into all aspects of drug development. This illustrates a hypothetical scenario of repurposing an existing drug for a new indication. 


\section{Funding}

None.

\section{Conflict of Interest}

T.Q. has received investigator-initiated research funding from Bristol-Meyers Squibb/Pfizer Alliance and has been a speaker for Bayer, Bristol-Myers Squibb and Pfizer. D.L. has received investigator-initiated educational grants from Bristol-Myers Squibb and Boehringer Ingelheim; has been a speaker for Boehringer Ingelheim, Bayer and Bristol-Myers Squibb/Pfizer; and has consulted for Bristol-Myers Squibb, Bayer, Boehringer Ingelheim and Daiichi-Sankyo.

\section{References}

1 Lip GYH, Freedman B, De Caterina R, Potpara TS. Stroke prevention in atrial fibrillation: past, present and future. Thromb Haemost 2017;117:1230-1239

2 Abdul-Rahim AH, Wong J, McAlpine C, Young C, Quinn TJ. Associations with anticoagulation: a cross-sectional registry-based analysis of stroke survivors with atrial fibrillation. Heart 2014;100(07):557-562

3 Bertozzo G, Zoppellaro G, Granziera S, et al. Reasons for and consequences of vitamin $\mathrm{K}$ antagonist discontinuation in very elderly patients with non-valvular atrial fibrillation. J Thromb Haemost 2016;14(11):2124-2131

4 Broekhuizen K, Pothof A, de Craen AJ, Mooijaart SP. Characteristics of randomized controlled trials designed for elderly: a systematic review. PLoS One 2015;10(05):e0126709

5 Mazurek M, Huisman MV, Rothman KJ, et al; GLORIA-AF Investigators. Regional differences in antithrombotic treatment for atrial fibrillation: insights from the GLORIA-AF Phase II Registry. Thromb Haemost 2017;117(12):2376-2388
6 Li XS, Deitelzweig S, Keshishian A, et al. Effectiveness and safety of apixaban versus warfarin in non-valvular atrial fibrillation patients in "real-world" clinical practice. A propensity-matched analysis of 76,940 patients. Thromb Haemost 2017;117(06): 1072-1082

7 Hohnloser SH, Basic E, Hohmann C, Nabauer M. Effectiveness and safety of non-vitamin $\mathrm{K}$ oral anticoagulants in comparison to phenprocoumon: data from 61,000 patients with atrial fibrillation. Thromb Haemost 2018;118(03):526-538

8 Doubal FN, Ali M, Batty GD, et al. Big data and data repurposing using existing data to answer new questions in vascular dementia research. BMC Neurol 2017;17(01):72

9 Hohmann C, Hohnloser SH, Jacob J, Walker J, Baldus S, Pfister R. Non-vitamin K oral anticoagulants in comparison to phenprocoumon in geriatric and non-geriatric patients with nonvalvular atrial fibrillation. Thromb Haemost 2019;119(06): 971-980

10 Hohnloser SH, Basic E, Nabauer M. Changes in oral anticoagulation therapy over one year in 51,000 atrial fibrillation patients at risk for stroke. Thromb Haemost 2019;119(06):882-893

11 Field TS, Weijs B, Curcio A, et al. Incident atrial fibrillation, dementia, and the role of anticoagulation: apopulation-based cohort study. Thromb Haemost 2019;119(06):981-991

12 Moffitt P, Lane DA, Park H, O'Connell J, Quinn TJ. Thromboprophylaxis in atrial fibrillation and association with cognitive decline: systematic review. Age Ageing 2016;45(06): 767-775

13 Wilkinson T, Ly A, Schnier C, et al; UK Biobank Neurodegenerative Outcomes Group and Dementias Platform UK. Identifying dementia cases with routinely collected health data: a systematic review. Alzheimers Dement 2018;14(08):1038-1051

14 Wehling M, Collins R, Gil VM, et al. Appropriateness of oral anticoagulants for the long-term treatment of atrial fibrillation in older people: results of an evidence-based review and international consensus validation process (OAC-FORTA 2016). Drugs Aging 2017;34(07):499-507 